\title{
SPECTRUM LOCALIZATION OF REGULAR MATRIX POLYNOMIALS AND FUNCTIONS*
}

\author{
A.G. MAZKO ${ }^{\dagger}$
}

\begin{abstract}
This paper is devoted to the spectrum localization problem for regular matrix polynomials and functions. Sufficient conditions are proposed for spectrum placement in a wide class of regions bounded by analytical curves. These conditions generalize the known linear matrix inequalities (LMI) approaches to stability analysis and pole placement of polynomial matrices. In addition, a method of robust spectrum placement is developed in the form of the LMI systems for a parametric set of matrix polynomials.
\end{abstract}

Key words. Matrix polynomial, Eigenvalue, Spectrum localization, Linear matrix inequality, Robust stability.

AMS subject classifications. 5A18, 15A22, 26C10.

1. Introduction. Many theoretical and applied problems are associated with the analysis of spectral properties of matrix functions including polynomial matrices. If direct evaluation of the eigenvalues does not leads to desirable outcomes, there are problems of their estimation and localization with respect to certain regions of a complex plane. Numerous works are devoted to solving these problems (see, e.g., $[1]-[4])$.

In this paper, we study the problem of spectrum localization for matrix polynomials and functions. We establish sufficient conditions for checking location of all eigenvalues of regular matrix functions in specified regions of the complex plane. The results obtained essential complement and generalize the known approaches to pole placement of polynomial matrices reduced to the LMI technics (see, e.g., [5] and [3, Section 2.7]). Moreover, we expand the obtained results to a parametric set of matrix polynomials in the form of the LMI systems.

Notation: $\otimes, *$, and $T$ denote the matrix operations of the Kronecker product, complex conjugation and transposition, respectively; $I_{n}$ is the identity $n \times n$ matrix; $O_{n}$ and $O_{n m}$ denote the zero $n \times n$ and $n \times m$ matrix, respectively; the matrix inequalities $X>Y, X \geq Y$, and $X \succeq Y$ mean that the matrix $X-Y$ is po-

\footnotetext{
*Received by the editors January 4, 2010. Accepted for publication May 28, 2010. Handling Editor: Harm Bart.

${ }^{\dagger}$ Institute of Mathematics, Ukrainian Academy of Sciences, Kyiv, Ukraine (mazko@imath.kiev.ua).
} 
sitive definite, positive semidefinite, and nonzero positive semidefinite, respectively; $i(X)=\left\{i_{+}(X), i_{-}(X), i_{0}(X)\right\}$ is the inertia of the Hermitian matrix $X=X^{*}$ consisting of its numbers of positive $\left(i_{+}(X)\right)$, negative $\left(i_{-}(X)\right)$, and zero $\left(i_{0}(X)\right)$ eigenvalues (taking into account the multiplicities); $\lambda_{\max }(X)\left(\lambda_{\min }(X)\right)$ is the maximum (minimum) eigenvalue of the Hermitian matrix $X$.

2. Matrix function and analytical regions. Consider the regular matrix function

$$
F(\lambda)=\sum_{i=0}^{m} f_{i}(\lambda) A_{i}, \quad \operatorname{det} F(\lambda) \not \equiv 0, \quad \lambda \in \mathbb{C}^{1},
$$

where $A_{0}, \ldots, A_{m} \in \mathbb{C}^{n \times n}$ are $n \times n$ matrices. Let $\sigma(F)$ be a spectrum of the matrix function that consists of all eigenvalues. Suppose that the scalar functions $f_{0}(\lambda), \ldots, f_{m}(\lambda)$ are analytical in a neighbourhood of $\sigma(F)$ and let

$$
z_{m}(\lambda)=\left[f_{0}(\lambda), \ldots, f_{m}(\lambda)\right] \neq 0, \quad \lambda \in \sigma(F) .
$$

We study a location of the spectrum with respect to regions in the complex plane of the type

$$
\Omega=\left\{\lambda \in \mathbb{C}^{1}: i_{+}(V(\lambda, \bar{\lambda})) \geq 1\right\}, \quad \hat{\Omega}=\left\{\lambda \in \mathbb{C}^{1}: V(\lambda, \bar{\lambda}) \leq 0\right\},
$$

where $V(\lambda, \bar{\lambda})$ is a Hermitian matrix-valued function. It is obvious that $\Omega \cap \hat{\Omega}=\emptyset$ and $\Omega \cup \hat{\Omega}=\mathbb{C}^{1}$.

Let's introduce the block matrices

$$
A=\left[\begin{array}{c}
A_{0} \\
\vdots \\
A_{m}
\end{array}\right], \quad G=\left[\begin{array}{c}
G_{0} \\
\vdots \\
G_{m}
\end{array}\right], \quad L=\left[\begin{array}{ccc}
L_{00} & \cdots & L_{0 m} \\
\vdots & \ddots & \vdots \\
L_{m 0} & \cdots & L_{m m}
\end{array}\right], \quad W=\left[\begin{array}{cc}
L & G \\
G^{*} & H
\end{array}\right] \text {. }
$$

Here $A_{i}, G_{i}, L_{i j}, H$ are the $n \times n$ blocks, and the matrices $L$ and $W$ are Hermitian.

We define the matrix $A^{\perp}=\left[B_{0}, \ldots, B_{m}\right]^{*}$ of size $n(m+1) \times n m$ as a basis for the right null space of $A^{*}$. Then

$$
A^{\perp *} A=0, \quad A^{+} A=I_{n}, \quad \operatorname{det} T \neq 0, \quad T=\left[A^{+*}, A^{\perp}\right],
$$

where $A^{+}=\left(A^{*} A\right)^{-1} A^{*}$ is the pseudoinverse matrix. Under regularity condition of the matrix function $F(\lambda)$, it is always possible to construct the matrix $A^{\perp}$ with specified properties.

Let the regions (2.2) be defined by

$$
V(\lambda, \bar{\lambda})=\sum_{i, j=0}^{m} f_{i}(\lambda) \overline{f_{j}(\lambda)} L_{i j}
$$


Lemma 2.1. If

$$
\left[I_{n(m+1)}, A\right] W\left[I_{n(m+1)}, A\right]^{*}>0
$$

or

$$
A^{\perp *} L A^{\perp}>0
$$

then $\sigma(F) \cap \hat{\Omega}=\emptyset$, i.e. all the eigenvalues of $F(\lambda)$ lie within $\Omega$.

Proof. It is obvious that $F(\lambda)=Z_{m}(\lambda) A$ and $V(\lambda, \bar{\lambda})=Z_{m}(\lambda) L Z_{m}^{*}(\lambda)$, where $Z_{m}(\lambda)=\left[f_{0}(\lambda) I_{n}, \ldots, f_{m}(\lambda) I_{n}\right]=z_{m}(\lambda) \otimes I_{n}$. We multiply $(2.4)$ on the left and on the right by $Z_{m}(\lambda)$ and $Z_{m}^{*}(\lambda)$, respectively. Then we obtain

$$
F(\lambda) G^{*}(\lambda)+G(\lambda) F^{*}(\lambda)+F(\lambda) H F^{*}(\lambda)+V(\lambda, \bar{\lambda})>0,
$$

where $G(\lambda)$ is a matrix function. Let $v_{0}^{*} \neq 0$ be the left eigenvector of the matrix function $F(\lambda)$ corresponding to an eigenvalue $\lambda_{0} \in \sigma(F)$. Multiplying the matrix inequality on the left and on the right by $v_{0}^{*}$ and $v_{0}$, respectively, at $\lambda=\lambda_{0}$, we have $v_{0}^{*} V\left(\lambda_{0}, \bar{\lambda}_{0}\right) v_{0}>0$, that means $\lambda_{0} \in \Omega$.

Let's multiply the matrix inequality (2.4) on the left and on the right by $T^{*}$ and $T$, respectively, and use the known criterion of positive definiteness of a block matrix:

$$
\left[\begin{array}{cc}
P & N \\
N^{*} & Q
\end{array}\right]>0 \Longleftrightarrow Q>0, \quad P>N Q^{-1} N^{*}
$$

In this case,

$$
P=H+G^{*} A^{+*}+A^{+} G+A^{+} L A^{+*}, \quad Q=A^{\perp *} L A^{\perp}, \quad N=\left(G^{*}+A^{+} L\right) A^{\perp} .
$$

Since last inequality in (2.6) may be always satisfied by choosing $H>0$, for inequality performance $(2.4)$, it is enough to demand that $Q>0$. Therefore, the inclusion $\sigma(F) \subset \Omega$ also follows from (2.5).

Note that, in Lemma 2.1, it is necessary to have the inequalities

$$
n(m+1) \leq i_{+}(W)<n(m+2), \quad 1 \leq i_{+}(L)<n(m+1) .
$$

As $H$, there may be any positive definite matrix, for example, $H=I_{n}$. If the inequality (2.4) holds for $H \leq 0$, then all the eigenvalues of the matrix function $G(\lambda)$ as well as the $F(\lambda)$ should belong to $\Omega$.

Supposing in Lemma 2.1 that $L=\Gamma \otimes X$ with the Hermitian matrices $\Gamma$ and $X$ of sizes $(m+1) \times(m+1)$ and $n \times n$, respectively, we obtain conditions when $\sigma(F)$ belongs to a region $\Omega$ described by the scalar Hermitian function $f(\lambda, \bar{\lambda})=z_{m}(\lambda) \Gamma z_{m}^{*}(\lambda)$. 
TheOREM 2.2. If

$$
A G^{*}+G A^{*}+A H A^{*}+\Gamma \otimes X>0, \quad X \succeq 0 ;
$$

or

$$
\sum_{i, j=0}^{m} \gamma_{i j} B_{i} X B_{j}^{*}>0, \quad X \succeq 0 ;
$$

then all the eigenvalues of the matrix function $F(\lambda)$ lie within the region

$$
\Omega=\left\{\lambda \in \mathbb{C}^{1}: f(\lambda, \bar{\lambda})=\sum_{i, j=0}^{m} \gamma_{i j} f_{i}(\lambda) \overline{f_{i}(\lambda)}>0\right\} .
$$

Let $f_{0}(\lambda) \neq 0$ in a neighbourhood of $\sigma(F)$, and let regions $\Omega$ and $\hat{\Omega}$ be defined as in $(2.2)$ with

$V(\lambda, \bar{\lambda})=\left[\begin{array}{cc}\Psi^{*}(\lambda) L \Psi(\lambda) & f_{0}(\lambda) \Psi^{*}(\lambda) G \\ \overline{f_{0}(\lambda)} G^{*} \Psi(\lambda) & f_{0}(\lambda) \overline{f_{0}(\lambda)} H\end{array}\right], \Psi(\lambda)=\left[\begin{array}{ccc}-f_{1}(\lambda) I_{n} & \cdots & -f_{m}(\lambda) I_{n} \\ f_{0}(\lambda) I_{n} & \cdots & O_{n} \\ \vdots & \ddots & \vdots \\ O_{n} & \cdots & f_{0}(\lambda) I_{n}\end{array}\right]$.

\section{Lemma 2.3. If}

$$
\left[A^{*}, I_{n}\right] W\left[A^{*}, I_{n}\right]^{*}>0,
$$

then $\sigma(F) \cap \hat{\Omega}=\emptyset$, i.e. all the eigenvalues of $F(\lambda)$ lie within $\Omega$.

Proof. Let $v_{0} \neq 0$ be the right eigenvector of the matrix function $F(\lambda)$ corresponding to an eigenvalue $\lambda_{0} \in \sigma(F)$. Multiplying (2.9) on the left and on the right by $\overline{f_{0}\left(\lambda_{0}\right)} v_{0}^{*}$ and $f_{0}\left(\lambda_{0}\right) v_{0}$, respectively, and taking into account the relations

$$
f_{0}\left(\lambda_{0}\right) A_{0} v_{0}=-\sum_{i=1}^{m} f_{i}\left(\lambda_{0}\right) A_{i} v_{0}, \quad w_{0}^{*}=\left[v_{0}^{*} A_{1}^{*}, \ldots, v_{0}^{*} A_{m}^{*}, v_{0}^{*}\right] \neq 0,
$$

we have $w_{0}^{*} V\left(\lambda_{0}, \bar{\lambda}_{0}\right) w_{0}>0$, that means $\lambda_{0} \in \Omega$.

Note that, in Lemma 2.3, it is necessary the inequality $n \leq i_{+}(W)<n(m+2)$.

Theorem 2.4. If

$$
\sum_{i, j=0}^{m} \gamma_{i j} A_{i}^{*} X A_{j}>0, \quad X \succeq 0
$$


then all the eigenvalues of the matrix function $F(\lambda)$ lie within the region

$$
\Omega=\left\{\lambda \in \mathbb{C}^{1}: i_{+}(U(\lambda, \bar{\lambda})) \geq 1\right\}
$$

where

$$
U(\lambda, \bar{\lambda})=\Phi^{*}(\lambda) \Gamma \Phi(\lambda), \quad \Phi(\lambda)=\left[\begin{array}{c}
-z(\lambda) \\
f_{0}(\lambda) I_{m}
\end{array}\right], \quad z(\lambda)=\left[f_{1}(\lambda), \ldots, f_{m}(\lambda)\right] .
$$

Theorem 2.4 follows from Lemma 2.3 when $G$ and $H$ are zero, and $L=\Gamma \otimes X$. Similar statement was formulated in [2] under weaker restrictions (controllability type) to the matrix expressions in (2.10). Lemmas 2.1 and 2.3 develop and complement the known approaches to pole placement of matrix functions (see [2, pp. 94-98] and [3, Section 2.7]) by means of the LMI (2.4), (2.5), (2.9) and their special cases (2.7), (2.8) and (2.10).

REMARK 2.5. If we choose $\Gamma$ in the form

$$
\Gamma=\left[\begin{array}{ccc}
0 & 1 & O_{1 m-1} \\
1 & q & O_{1 m-1} \\
O_{m-11} & O_{m-11} & -Q^{-1}
\end{array}\right], \quad Q=Q^{*}>0,
$$

then $\Omega$ in Theorem 2.4 can be described by the scalar function:

$$
\Omega=\left\{\lambda \in \mathbb{C}^{1}: f(\lambda, \bar{\lambda})=z_{m}(\lambda) \widetilde{\Gamma} z_{m}^{*}(\lambda)>0\right\}, \quad \widetilde{\Gamma}=\left[\begin{array}{ccc}
q & -1 & O_{1 m-1} \\
-1 & 0 & O_{1 m-1} \\
O_{m-11} & O_{m-11} & Q
\end{array}\right]
$$

EXAMPLE 2.6. Consider the regular matrix quasipolynomial

$$
F(\lambda)=A_{0}+\lambda A_{1}+e^{-\lambda \tau_{1}} A_{2}+\cdots+e^{-\lambda \tau_{m-1}} A_{m}, \quad \tau_{i} \geq 0, \quad i=\overline{1, m-1} .
$$

If any numbers $q, q_{1}>0, \ldots, q_{m-1}>0$ and matrix $X \succeq 0$ satisfy the matrix inequality

$$
A_{0}^{*} X A_{1}+A_{1}^{*} X A_{0}+q A_{1}^{*} X A_{1}-\sum_{i=1}^{m-1} \frac{1}{q_{i}} A_{i+1}^{*} X A_{i+1}>0,
$$

then according to Theorem 2.4 all the eigenvalues of $F(\lambda)$ lie within the region

$$
\Omega=\left\{\lambda \in \mathbb{C}^{1}: \lambda+\bar{\lambda}<q+\sum_{i=1}^{m-1} q_{i} e^{-(\lambda+\bar{\lambda}) \tau_{i}}\right\} .
$$

If $q \leq-q_{1}-\cdots-q_{m-1}$, then given region is located in the left half-plane. In this case, we have sufficient stability conditions of quasipolynomial $F(\lambda)$ in the form of LMI $(2.11)$ (see $[2,3])$. This result can be used in the absolute stability analysis of the linear time-delay control systems. 
3. Matrix polynomial and algebraic regions. Given the regular matrix polynomial of size $n \times n$ and degree $s$

$$
F(\lambda)=A_{0}+\lambda A_{1}+\cdots+\lambda^{s} A_{s}, \quad \operatorname{det} F(\lambda) \not \equiv 0, \quad \lambda \in \mathbb{C}^{1},
$$

we consider the class of algebraic regions:

$$
\Lambda_{k}=\left\{\lambda \in \mathbb{C}^{1}: f(\lambda, \bar{\lambda})=\sum_{i, j=0}^{k} \gamma_{i j} \lambda^{i} \bar{\lambda}^{j}>0\right\}
$$

where $\gamma_{i j}$ are entries of the Hermitian matrix $\Gamma$, and $s \geq 1, k \geq 1$. We assume that $\Lambda_{k} \neq \emptyset$ and $\Lambda_{k} \neq \mathbb{C}^{1}$, i.e. $i_{ \pm}(\Gamma) \neq 0$.

It is obvious that any region $\Lambda_{1}$ is bounded by a line or a circle. In particular, the matrices

$$
\Gamma=\left[\begin{array}{cc}
0 & -1 \\
-1 & 0
\end{array}\right], \quad \Gamma=\left[\begin{array}{cc}
1 & 0 \\
0 & -1
\end{array}\right]
$$

correspond to the left half-plane and the unit disk. The class $\Lambda_{2}$ contains all the regions bounded by algebraic curves of the second order.

Let $m=\max \{s, k\}$ and $r=m-k$. We construct the block matrices

$$
A=\left[\begin{array}{c}
A_{0} \\
\vdots \\
A_{m}
\end{array}\right], \quad G=\left[\begin{array}{c}
G_{0} \\
\vdots \\
G_{m}
\end{array}\right], \quad X=\left[\begin{array}{ccc}
X_{00} & \cdots & X_{0 r} \\
\vdots & \ddots & \vdots \\
X_{r 0} & \cdots & X_{r r}
\end{array}\right]
$$

of sizes $n(m+1) \times n, n(m+1) \times n$ and $n(r+1) \times n(r+1)$, respectively, and introduce the linear operators

$$
L(X)=C(\Gamma \otimes X) C^{T}, \quad M(X)=D(\Gamma \otimes X) D^{*},
$$

where

$$
\begin{gathered}
C=R \otimes I_{n}=\left[C_{0}, \ldots, C_{k}\right], \quad D=A^{\perp *} C=\left[D_{0}, \ldots, D_{k}\right], \\
R=\left[E, \Delta E, \ldots, \Delta^{k} E\right], \quad \Delta=\left[\begin{array}{cc}
O_{1 m} & 0 \\
I_{m} & O_{m 1}
\end{array}\right], \quad E=\left[\begin{array}{c}
I_{r+1} \\
O_{k r+1}
\end{array}\right],
\end{gathered}
$$

$A^{\perp *}=\left[B_{0}, \ldots, B_{m}\right]$ is the matrix defined by (2.3). In the case of $k>s$, the blocks $A_{i}$ $(i>s)$ should be chosen in such a way that the spectrum of the matrix polynomial $F_{m}(\lambda)=A_{0}+\lambda A_{1}+\cdots+\lambda^{m} A_{m}$ contains $\sigma(F)$. For example, we can suppose that $A_{i}=0$ for $i>s$. 
Let $\hat{\Lambda}_{k} \triangleq \mathbb{C}^{1} \backslash \Lambda_{k}$ be the closed complement of $\Lambda_{k}$, and let $\Lambda_{r}(X)$ be the closed region defined by the block Hermitian matrix $X$ :

$\Lambda_{r}(X)=\left\{\lambda \in \mathbb{C}^{1}: Z_{r}(\lambda) X Z_{r}^{*}(\lambda)=\sum_{i, j=0}^{r} \lambda^{i} \bar{\lambda}^{j} X_{i j} \geq 0\right\}, \quad Z_{r}(\lambda)=\left[I_{n}, \lambda I_{n}, \ldots, \lambda^{r} I_{n}\right]$.

TheOREM 3.1. Let for some matrices $G, H=H^{*}, X=X^{*}$, the inclusion

$$
\hat{\Lambda}_{k} \subseteq \Lambda_{r}(X)
$$

and one of the matrix inequalities

$$
A G^{*}+G A^{*}+A H A^{*}+L(X)>0
$$

or

$$
M(X)>0
$$

hold. Then all the eigenvalues of the matrix polynomial $F(\lambda)$ lie within the region $\Lambda_{k}$.

Proof. The matrix $R$ of size $(m+1) \times(k+1)(r+1)$ has the following structure:

$$
R=\left[E, \Delta E, \ldots, \Delta^{k} E\right]=\left[\begin{array}{c|c|c|c}
I_{r+1} & O_{1 r+1} & & O_{1 r+1} \\
O_{1 r+1} & I_{r+1} & \ldots & O_{k-1 r+1} \\
O_{k-1 r+1} & O_{k-1 r+1} & & I_{r+1}
\end{array}\right] .
$$

It is obvious that

$$
F_{m}(\lambda)=Z_{m}(\lambda) A, \quad f(\lambda, \bar{\lambda})=z_{k}(\lambda) \Gamma z_{k}^{*}(\lambda),
$$

where $Z_{m}(\lambda)=z_{m}(\lambda) \otimes I_{n}, z_{m}(\lambda)=\left[1, \lambda, \ldots, \lambda^{m}\right]$. Using the structure of matrix $C$ and the Kronecker products properties, we have

$$
\begin{gathered}
Z_{m}(\lambda) C=\left(z_{m}(\lambda) \otimes I_{n}\right)\left(R \otimes I_{n}\right)=z_{m}(\lambda) R \otimes I_{n}= \\
=\left[z_{r}(\lambda), \lambda z_{r}(\lambda), \ldots, \lambda^{k} z_{r}(\lambda)\right] \otimes I_{n}=z_{k}(\lambda) \otimes z_{r}(\lambda) \otimes I_{n}=z_{k}(\lambda) \otimes Z_{r}(\lambda) .
\end{gathered}
$$

Multiplying (3.6) on the left and on the right by the full rank matrices $Z_{m}(\lambda)$ and $Z_{m}^{*}(\lambda)$, respectively, we obtain

$$
\begin{gathered}
F_{m}(\lambda) G_{m}^{*}(\lambda)+G_{m}(\lambda) F_{m}^{*}(\lambda)+F_{m}(\lambda) H F_{m}^{*}(\lambda)+\left[z_{k}(\lambda) \otimes Z_{r}(\lambda)\right](\Gamma \otimes X)\left[z_{k}^{*}(\lambda) \otimes Z_{r}^{*}(\lambda)\right]= \\
=F_{m}(\lambda) G_{m}^{*}(\lambda)+G_{m}(\lambda) F_{m}^{*}(\lambda)+F_{m}(\lambda) H F_{m}^{*}(\lambda)+f(\lambda, \bar{\lambda}) Z_{r}(\lambda) X Z_{r}^{*}(\lambda)>0,
\end{gathered}
$$


where $G_{m}(\lambda)=G_{0}+\lambda G_{1}+\cdots+\lambda^{m} G_{m}$ is a matrix polynomial.

Let $\lambda=\lambda_{0} \in \sigma(F)$ be an arbitrary eigenvalue of $F(\lambda)$. Also we assume that $\lambda_{0} \notin \Lambda_{k}$, i.e. $\lambda_{0} \in \hat{\Lambda}_{k}$. Then, according to (3.5), $f\left(\lambda_{0}, \bar{\lambda}_{0}\right) Z_{r}\left(\lambda_{0}\right) X Z_{r}^{*}\left(\lambda_{0}\right) \leq 0$. Thus,

$$
F_{m}\left(\lambda_{0}\right) G_{m}^{*}\left(\lambda_{0}\right)+G_{m}\left(\lambda_{0}\right) F_{m}^{*}\left(\lambda_{0}\right)+F_{m}\left(\lambda_{0}\right) H F_{m}^{*}\left(\lambda_{0}\right)>0 .
$$

However, this is not true, because $\sigma(F) \subseteq \sigma\left(F_{m}\right)$ and

$$
v_{0}^{*}\left[F_{m}\left(\lambda_{0}\right) G_{m}^{*}\left(\lambda_{0}\right)+G_{m}\left(\lambda_{0}\right) F_{m}^{*}\left(\lambda_{0}\right)+F_{m}\left(\lambda_{0}\right) H F_{m}^{*}\left(\lambda_{0}\right)\right] v_{0}=0,
$$

where $v_{0}^{*} \neq 0$ is the left eigenvector of the matrix polynomial $F_{m}(\lambda)$ corresponding to $\lambda_{0} \in \sigma\left(F_{m}\right)$. From this contradiction, it follows that, under conditions (3.5) and (3.6), it should be $\lambda_{0} \in \Lambda_{k}$.

From (3.5) and (3.7), it also follows that $\sigma(F) \subset \Omega$. It is established by multiplying (3.6) on the left and on the right by $T$ and $T^{*}$, respectively, and applying the criterion (2.6) (see the proof of Lemma 2.1).

Theorem 3.1 develops known approach for the spectrum localization of a matrix polynomial proposed in [5] for the class of regions $\Lambda_{1}$. We do not use any restrictions to the order of the algebraic curves. In addition, condition (3.5) generally does not demand the positive definiteness of solutions of the corresponding matrix inequalities. The results obtained in [4] extend the approach of [5] by considering algebraic regions $\mathcal{D}$ described by the quadratic matrix inequalities and involving the optimization algorithms. Every $\mathcal{D}$-region can always be written as a finite set of $\Omega$-regions. However, the converse procedure is nontrivial and unsolved problem for the present.

REMARK 3.2. The proof of Theorem 3.1 can be obtained as a corollary of Lemma 2.1. Indeed, in the case of the matrix polynomial, we suppose that $f_{i}(\lambda)=\lambda^{i}$ for $i=$ $\overline{0, m}$, and, in virtue of (3.5), $\Omega \subseteq \Lambda_{k}$. The restriction (3.5) holds for arbitrary region $\Lambda_{k}$ when $\Lambda_{r}(X)=\mathbb{C}^{1}$. For example, we can find $X$ as an algebraically nonnegative defined matrix, in particular, nonnegative defined matrix in usual sense.

Definition 3.3. The block Hermitian matrix $X$ is called algebraically positive (nonnegative) defined if

$$
Z_{r}(\lambda) X Z_{r}^{*}(\lambda)=\sum_{i, j=0}^{r} \lambda^{i} \bar{\lambda}^{j} X_{i j}>0(\geq 0), \forall \lambda \in \mathbb{C}^{1} .
$$

Note that all the matrices of the type

$$
X=\left[\begin{array}{cc}
X_{p} & O_{n(p+1) n(r-p)} \\
O_{n(r-p) n(p+1)} & O_{n(r-p) n(r-p)}
\end{array}\right], \quad X_{p}=\left[\begin{array}{ccc}
X_{00} & \cdots & X_{0 p} \\
\vdots & \ddots & \vdots \\
X_{p 0} & \cdots & X_{p p}
\end{array}\right]>0,
$$


are algebraically positive defined. These matrices are positive defined when $p=r$.

REMARK 3.4. If $k \geq s$, then $m=k, r=0$, and $C=I_{n(k+1)}$. In this case, in (3.6) and (3.7), we suppose $L(X)=\Gamma \otimes X$ and $M(X)=A^{\perp *} L(X) A^{\perp}$. In addition, in Theorem 3.1, we use the inequality $X=X^{*} \succeq 0$ instead of (3.5).

REMARK 3.5. Under certain restrictions, the proposed sufficient conditions for spectrum inclusion $\sigma(F) \subset \Lambda_{k}$ can become necessary conditions. If the LMI (3.6) holds, then $i_{+}(L(X)) \geq n m$, and consequently (see [3, Theorem 4.2.1]),

$$
i_{+}(\Gamma) i_{+}(X)+i_{-}(\Gamma) i_{-}(X) \geq n m .
$$

This inequality is necessary also for feasibility of the LMI (3.7). Particularly, in the case of $k \geq s$ and $X>0$, it is necessary that $i(\Gamma)=\{k, 1,0\}$. The necessary and sufficient solvability conditions of (3.7) can be studied via the general theorems on inertia of Hermitian solutions of transformable matrix equations [2, 3]. Especially, such conditions are connected with constraints for the inertia $i(\Gamma)$ and the property of simultaneous reducibility of the matrix coefficients $D_{i}=A^{\perp *} C_{i}(i=\overline{0, k})$ to triangular form through common similarity transformation. When $k=1$, the matrices $D_{i}$ are quadratic (see, e.g., Theorem 3.7 and Example 3.8 below).

Notice that if $\operatorname{det} A_{0} \neq 0$, then in (2.3), $A^{\perp *}$ always can be chosen as

$$
A^{\perp *}=\left[\begin{array}{cccc}
\hat{A}_{1} & I_{n} & \cdots & O_{n} \\
\vdots & \vdots & \ddots & \vdots \\
\hat{A}_{m} & O_{n} & \cdots & I_{n}
\end{array}\right], \quad \hat{A}_{i}=\left\{\begin{array}{cc}
-A_{i} A_{0}^{-1}, & i \leq s, \\
O_{n}, & i>s .
\end{array}\right.
$$

When $k=s$, the operator $M(X)$ in (3.7), in virtue of (3.8), has the block structure:

$$
M(X)=\left[\begin{array}{ccc}
Y_{11}(Z) & \cdots & Y_{1 s}(Z) \\
\vdots & \ddots & \vdots \\
Y_{s 1}(Z) & \cdots & Y_{s s}(Z)
\end{array}\right], \quad X=A_{0} Z A_{0}^{*},
$$

where $Y_{p q}(Z)=\gamma_{00} A_{p} Z A_{q}^{*}-\gamma_{p 0} A_{0} Z A_{q}^{*}-\gamma_{0 q} A_{p} Z A_{0}^{*}+\gamma_{p q} A_{0} Z A_{0}^{*}, p, q=\overline{1, s}$.

If $\operatorname{det} A_{0}=0$, then we consider, instead of $F(\lambda)$ and $\Lambda_{k}$, the matrix polynomial and region, respectively, as follows:

$F_{\alpha}(\lambda)=F(\lambda+\alpha)=\sum_{i=0}^{s} \lambda^{i} A_{\alpha i}, \quad \Lambda_{k}^{\alpha}=\left\{\lambda \in \mathbb{C}^{1}: f(\lambda+\alpha, \bar{\lambda}+\bar{\alpha})=\sum_{i, j=0}^{k} \gamma_{i j}^{\alpha} \lambda^{i} \bar{\lambda}^{j}>0\right\}$,

where $A_{\alpha 0}=F(\alpha), \operatorname{det} A_{\alpha 0} \neq 0$. Since $F(\lambda)$ is a regular matrix polynomial, there is $\alpha \notin \sigma(F)$ with specified properties. Thus, $\sigma(F) \subset \Lambda_{k} \Longleftrightarrow \sigma\left(F_{\alpha}\right) \subset \Lambda_{k}^{\alpha}$, and $\sigma\left(F_{\alpha}\right)=\sigma(F)-\alpha$. 
There are various methods of reduction of spectral problems for matrix polynomials to similar problems for linear pencils of matrices (see, for example, [6]-[9]). We use the method [9] based on applying the matrices of type $A^{\perp}$ satisfying (2.3). Using the block representation $A^{\perp *}=\left[B_{0}, \ldots, B_{s}\right]$, we construct the linear pencil of matrices

$$
D_{1}-\lambda D_{0}=\left[B_{1}, \ldots, B_{s}\right]-\lambda\left[B_{0}, \ldots, B_{s-1}\right]
$$

and consider the following relations

$$
\begin{gathered}
v^{*} F(\lambda)=0, \quad v \neq 0 ; \\
u^{*}\left(D_{1}-\lambda D_{0}\right)=0, \quad u \neq 0 ; \\
u^{*} A^{\perp *}=v^{*} Z_{s}(\lambda), \quad v=B_{0}^{*} u \neq 0 .
\end{gathered}
$$

The relations (3.11) and (3.12) define the eigenvalues and the corresponding left eigenvectors of matrix polynomial (3.1) and pencil (3.10), respectively.

It is easy to establish equivalence of (3.12) and (3.13). On the other hand, from definition of $A^{\perp}$ and representations $F(\lambda)=Z_{s}(\lambda) A$, it follows that (3.11) is equivalent to (3.13) for certain vector $u \neq 0$. Hence, (3.11) and (3.12) are equivalent. Additionally, identical fulfilment of one of equalities (3.11) or (3.12) at every $\lambda \in \mathbb{C}^{1}$ implies identical fulfilment of other of them. This means that regularity properties of matrix polynomial (3.1) and pencil (3.10) are equivalent, too.

Lemma 3.6. Sets of all the various eigenvalues $\lambda_{i}$ of matrix polynomial (3.1) and pencil (3.10) coincide, and their corresponding left eigenvectors are related by $v_{i}^{*}=u_{i}^{*} B_{0}(i=\overline{1, l})$.

Now we formulate criteria of inclusion $\sigma(F) \subset \Lambda_{1}$. In this case,

$$
\begin{gathered}
E=\left[\begin{array}{c}
I_{s} \\
O_{1 s}
\end{array}\right], \quad \Delta=\left[\begin{array}{cc}
O_{1 s} & 0 \\
I_{s} & O_{s 1}
\end{array}\right], \\
M(X)=\gamma_{00} D_{0} X D_{0}^{*}+\gamma_{01} D_{0} X D_{1}^{*}+\gamma_{10} D_{1} X D_{0}^{*}+\gamma_{11} D_{1} X D_{1}^{*},
\end{gathered}
$$

where $D_{0}$ and $D_{1}$ are matrices defined in (3.10). In particular, for matrix (3.8), we have

$$
D_{0}=\left[\begin{array}{cccc}
\hat{A}_{1} & I_{n} & \cdots & O_{n} \\
\vdots & \vdots & \ddots & \vdots \\
\hat{A}_{s-1} & O_{n} & \cdots & I_{n} \\
\hat{A}_{s} & O_{n} & \cdots & O_{n}
\end{array}\right], \quad D_{1}=I_{n s} .
$$


Considering Lemma 3.6 and general properties of positive invertible operators in the space of the Hermitian matrices, it is possible to formulate the following statement (see $[2,3])$.

THEOREM 3.7. The inclusion $\sigma(F) \subset \Lambda_{1}$ holds if and only if there exist Hermitian matrices $X$ and $Y$ satisfying the relations

(a) $M(X)=Y \geq 0, \quad D_{0} X D_{0}^{*} \geq 0, \quad \operatorname{rank}\left[D_{1}-\lambda D_{0}, Y\right] \equiv n s\left(\lambda \in \hat{\Lambda}_{1}\right)$.

If $D_{0}$ is nonsingular, then (a) is equivalent to each of the following statements:

(b) the matrix inequality $M(X)>0$ has a solution $X>0$;

(c) given any $Y>0$, the matrix equation $M(X)=Y$ has a solution $X>0$;

(d) operator $M$ is positive invertible with respect to a cone of nonnegative definite matrices.

The proof of sufficiency of the criterion (a) consists in the following. If we assume that some eigenvalue $\lambda \in \hat{\Lambda}_{1}$, then, according to (3.12), for the corresponding left eigenvector $u^{*}$, the inconsistent relations should be held true:

$$
f(\lambda, \bar{\lambda}) \leq 0, \quad u^{*} D_{0} X D_{0}^{*} u \geq 0, \quad f(\lambda, \bar{\lambda}) u^{*} D_{0} X D_{0}^{*} u=u^{*} Y u>0 .
$$

Provided that $\sigma(F) \subset \Lambda_{1}$, the matrices $X$ and $Y$ in criterion (a) always can be constructed in the form [2]

$$
X=Z \hat{X} Z^{*}, \quad Y=D_{0} Z \hat{Y} Z^{*} D_{0}^{*}, \quad \operatorname{rank}\left[D_{1} Z, D_{0} Z\right]=\operatorname{rank}\left(D_{0} Z\right) .
$$

This fact is established using the Kronecker canonical form of a regular matrix pencil [11]. If $D_{0}$ is nonsingular, then the criteria (b), (c) and (d) follow from known theorems on localization of eigenvalues by means of the generalized Lyapunov equation.

ExAmPle 3.8. Let $F(\lambda)=A_{0}+\lambda A_{1}$ be a regular pencil of $n \times n$ matrices, and $\Lambda_{1}$ be a region of the form (3.2). The matrix inequality (3.6) in Theorem 3.1 may be expressed as

$$
\left[\begin{array}{cc}
A_{0} H A_{0}^{*}+A_{0} G_{0}^{*}+G_{0} A_{0}^{*}+\gamma_{00} X & A_{0} H A_{1}^{*}+A_{0} G_{1}^{*}+G_{0} A_{1}^{*}+\gamma_{01} X \\
A_{1} H A_{0}^{*}+A_{1} G_{0}^{*}+G_{1} A_{0}^{*}+\gamma_{10} X & A_{1} H A_{1}^{*}+A_{1} G_{1}^{*}+G_{1} A_{1}^{*}+\gamma_{11} X
\end{array}\right]>0 .
$$

The matrix inequality (3.7) constructed for the linear pencil $F_{\alpha}(\lambda)=F(\alpha)+\lambda A_{1}$ and the regions $\Lambda_{1}^{\alpha}=\left\{\lambda \in \mathbb{C}^{1}: f(\lambda+\alpha, \bar{\lambda}+\bar{\alpha})>0\right\}$ in virtue of $A^{\perp *}=\left[B_{0}, B_{1}\right]=$ $\left[-A_{1} F^{-1}(\alpha), I_{n}\right]$ is reduced to the form

$$
M(X)=\gamma_{00} A_{1} Z A_{1}^{*}-\gamma_{10} A_{0} Z A_{1}^{*}-\gamma_{01} A_{1} Z A_{0}^{*}+\gamma_{11} A_{0} Z A_{0}^{*}>0,
$$

where $X=F(\alpha) Z F^{*}(\alpha), \alpha \notin \sigma(F)$. The given inequality due to equivalence of the inclusions $\sigma(F) \subset \Lambda_{1}$ and $\sigma\left(F_{\alpha}\right) \subset \Lambda_{1}^{\alpha}$, we use in Theorems 3.1 and 3.7 for initial 
matrix pencil $F(\lambda)$ and regions $\Lambda_{1}$. In particular, existence of solutions $Z=Z^{*}>0$ of the matrix inequalities

$$
A_{0} Z A_{1}^{*}+A_{1} Z A_{0}^{*}>0, \quad A_{1} Z A_{1}^{*}-A_{0} Z A_{0}^{*}>0
$$

is equivalent to location of the spectrum $\sigma(F)$ inside the left half-plane and the unit disk, respectively.

ExAmple 3.9. Let $F(\lambda)=A_{0}+\lambda A_{1}+\lambda^{2} A_{2}$ be a quadratic pencil of $n \times n$ matrices, and $\Lambda_{k}$ be a region of the type (3.2) for $k \leq 2$. Then the expression $A G^{*}+G A^{*}+A H A^{*}$ in (3.6) has the block form

$$
\left[\begin{array}{ccc}
A_{0} G_{0}^{*}+G_{0} A_{0}^{*}+A_{0} H A_{0}^{*} & A_{0} G_{1}^{*}+G_{0} A_{1}^{*}+A_{0} H A_{1}^{*} & A_{0} G_{2}^{*}+G_{0} A_{2}^{*}+A_{0} H A_{2}^{*} \\
A_{1} G_{0}^{*}+G_{1} A_{0}^{*}+A_{1} H A_{0}^{*} & A_{1} G_{1}^{*}+G_{1} A_{1}^{*}+A_{1} H A_{1}^{*} & A_{1} G_{2}^{*}+G_{1} A_{2}^{*}+A_{1} H A_{2}^{*} \\
A_{2} G_{0}^{*}+G_{2} A_{0}^{*}+A_{2} H A_{0}^{*} & A_{2} G_{1}^{*}+G_{2} A_{1}^{*}+A_{2} H A_{1}^{*} & A_{2} G_{2}^{*}+G_{2} A_{2}^{*}+A_{2} H A_{2}^{*}
\end{array}\right] .
$$

For the class of regions $\Lambda_{1}$, we have $1=k<s=2, m=2, r=1$,

$$
\begin{aligned}
& E=\left[\begin{array}{ll}
1 & 0 \\
0 & 1 \\
0 & 0
\end{array}\right], \Delta=\left[\begin{array}{lll}
0 & 0 & 0 \\
1 & 0 & 0 \\
0 & 1 & 0
\end{array}\right], R=\left[\begin{array}{llll}
1 & 0 & 0 & 0 \\
0 & 1 & 1 & 0 \\
0 & 0 & 0 & 1
\end{array}\right], \quad X=\left[\begin{array}{ll}
X_{00} & X_{01} \\
X_{10} & X_{11}
\end{array}\right] \text {, } \\
& L(X)=\left[\begin{array}{ccc}
\gamma_{00} X_{00} & \gamma_{00} X_{01}+\gamma_{01} X_{00} & \gamma_{01} X_{01} \\
\gamma_{00} X_{10}+\gamma_{10} X_{00} & \sum_{i, j=0}^{1} \gamma_{i j} X_{1-i 1-j} & \gamma_{01} X_{11}+\gamma_{11} X_{01} \\
\gamma_{10} X_{10} & \gamma_{10} X_{11}+\gamma_{11} X_{10} & \gamma_{11} X_{11}
\end{array}\right]
\end{aligned}
$$

For the class of regions $\Lambda_{2}$, in (3.6) and (3.9), we use the operators

$$
L(X)=\left[\begin{array}{ccc}
\gamma_{00} X & \gamma_{01} X & \gamma_{02} X \\
\gamma_{10} X & \gamma_{11} X & \gamma_{12} X \\
\gamma_{20} X & \gamma_{21} X & \gamma_{22} X
\end{array}\right], \quad M(X)=\left[\begin{array}{cc}
Y_{11}(Z) & Y_{12}(Z) \\
Y_{21}(Z) & Y_{22}(Z)
\end{array}\right]
$$

where

$$
\begin{aligned}
& Y_{11}(Z)=\gamma_{00} A_{1} Z A_{1}^{*}-\gamma_{10} A_{0} Z A_{1}^{*}-\gamma_{01} A_{1} Z A_{0}^{*}+\gamma_{11} A_{0} Z A_{0}^{*}, \\
& Y_{12}(Z)=\gamma_{00} A_{1} Z A_{2}^{*}-\gamma_{10} A_{0} Z A_{2}^{*}-\gamma_{02} A_{1} Z A_{0}^{*}+\gamma_{12} A_{0} Z A_{0}^{*}, \\
& Y_{22}(Z)=\gamma_{00} A_{2} Z A_{2}^{*}-\gamma_{20} A_{0} Z A_{2}^{*}-\gamma_{02} A_{2} Z A_{0}^{*}+\gamma_{22} A_{0} Z A_{0}^{*} .
\end{aligned}
$$

EXAMPLE 3.10. Let $F(\lambda)=a_{0}+\lambda a_{1}+\cdots+\lambda^{s} a_{s}$ be a scalar polynomial of degree $s$. The matrix inequality (3.6) has the form

$$
a g^{*}+g a^{*}+h a a^{*}+L(X)>0,
$$


where $a=\left[a_{0}, \ldots, a_{s}\right]^{*}, h \in \mathbb{R}^{1}, g \in \mathbb{C}^{s+1}$. Specifying $L(X)$ for the class of regions $\Lambda_{1}$, we use the matrices

$$
E=\left[\begin{array}{c}
I_{s} \\
O_{1 s}
\end{array}\right], \quad \Delta=\left[\begin{array}{cc}
O_{1 s} & 0 \\
I_{s} & O_{s 1}
\end{array}\right], \quad R=\left[\begin{array}{c|c}
I_{s} & O_{1 s} \\
O_{1 s} & I_{s}
\end{array}\right], \quad X=\left\|x_{i j}\right\|_{i, j=0}^{s-1} .
$$

In particular, for the left half-plane and the unit disk defined by corresponding matrices (3.3), we have

$$
L(X)=-\left[\begin{array}{ccccc}
0 & x_{00} & \cdots & x_{0 s-2} & x_{0 s-1} \\
x_{00} & x_{01}+x_{10} & \cdots & x_{0 s-1}+x_{1 s-2} & x_{1 s-1} \\
\vdots & \vdots & \ddots & \vdots & \vdots \\
x_{s-20} & x_{s-10}+x_{s-21} & \cdots & x_{s-1 s-2}+x_{s-2 s-1} & x_{s-1 s-1} \\
x_{s-10} & x_{s-11} & \cdots & x_{s-1 s-1} & 0
\end{array}\right]
$$

and

$$
L(X)=\left[\begin{array}{ccccc}
x_{00} & x_{01} & \cdots & x_{0 s-1} & 0 \\
x_{10} & x_{11}-x_{00} & \cdots & x_{1 s-1}-x_{0 s-2} & -x_{0 s-1} \\
\vdots & \vdots & \ddots & \vdots & \vdots \\
x_{s-10} & x_{s-11}-x_{s-20} & \cdots & x_{s-1 s-1}-x_{s-2 s-2} & -x_{s-2 s-1} \\
0 & -x_{s-10} & \cdots & -x_{s-1 s-2} & -x_{s-1 s-1}
\end{array}\right]
$$

The operator $L(X)=x \Gamma$ is a matrix-valued function of scalar argument $X=x$ for any region $\Lambda_{k}$ at $k \geq s$.

Note that in [10, Theorem 1], it is obtained a LMI characterization of the roots of polynomials inclusion into regions $\Lambda_{1}$, which is similar to (3.15) with other form of the operator $L(X)$.

4. Robust spectrum localization. In applications, the robust stability and the robust spectrum localization problems formulated for dynamic systems with parametric uncertainty are very important (see, for example, [12]-[14]). Solving these problems, there can be useful results formulated above for matrix functions.

As an example, we consider the parametric set of regular matrix polynomials:

$$
F(\lambda, p)=A_{0}\left(p_{0}\right)+\lambda A_{1}\left(p_{1}\right)+\cdots+\lambda^{s} A_{s}\left(p_{s}\right), \quad \operatorname{det} F(\lambda, p) \not \equiv 0, \lambda \in \mathbb{C}^{1},
$$

The values of coefficient matrices $A_{i}\left(p_{i}\right)$ depending on vector parameters

$$
p_{i}=\left[p_{i 1}, \ldots, p_{i \nu_{i}}\right]^{T} \in \mathcal{P}_{\nu_{i}} \triangleq\left\{q \in \mathbb{R}^{\nu_{i}}: q_{1} \geq 0, \ldots, q_{\nu_{i}} \geq 0, \sum_{j=1}^{\nu_{i}} q_{j}=1\right\},
$$


constitute a set of the matrix polytopes

$$
A_{i}=\left\{A \in \mathbb{C}^{n \times n}: A=\sum_{j=1}^{\nu_{i}} p_{i j} A_{i j}, \quad p_{i} \in \mathcal{P}_{\nu_{i}}\right\}, \quad i=\overline{0, s} .
$$

The general vector of parameters $p=\left[p_{0}^{T}, \ldots, p_{s}^{T}\right]^{T} \in \mathcal{P}=\mathcal{P}_{\nu_{0}} \times \cdots \times \mathcal{P}_{\nu_{s}}$ has order $\nu=\nu_{0}+\cdots+\nu_{s}$.

We specify all the $\nu_{0} \cdots \nu_{s}$ polynomial matrix vertices as follows:

$$
F_{t_{0} \cdots t_{s}}(\lambda)=A_{0 t_{0}}+\lambda A_{1 t_{1}}+\cdots+\lambda^{s} A_{s t_{s}}, \quad t_{i} \in\left\{1, \ldots, \nu_{i}\right\}, \quad i=\overline{0, s} .
$$

If $\nu_{i}=1$ for some $i$, then $t_{i}=1$, and $A_{i}$ in (4.1) does not depend on $p_{i}$.

Lemma 4.1. If $p \in \mathcal{P}_{\nu}$, then $p p^{T} \leq P=\operatorname{diag}\left\{p_{1}, \ldots, p_{\nu}\right\}$.

Proof. For any vector $x \in R^{\nu}$, we have

$$
\begin{aligned}
x^{T}\left(P-p p^{T}\right) x & =\sum_{i=1}^{\nu} p_{i} x_{i}^{2}-\left(\sum_{i=1}^{\nu} p_{i} x_{i}\right)^{2}=\left(\sum_{i=1}^{\nu} p_{i} x_{i}^{2}\right)\left(\sum_{j=1}^{\nu} p_{j}\right)-\left(\sum_{i=1}^{\nu} p_{i} x_{i}\right)^{2}= \\
& =\sum_{i \neq j} p_{i} p_{j} x_{i}^{2}-\sum_{i \neq j} p_{i} p_{j} x_{i} x_{j}=\sum_{i<j} p_{i} p_{j}\left(x_{i}-x_{j}\right)^{2} \geq 0 .
\end{aligned}
$$

Hence, $p p^{T} \leq P$.

Theorem 4.2. Let $G, H=H^{*} \leq 0$ and $X_{t_{0} \cdots t_{s}}=X_{t_{0} \cdots t_{s}}^{*}$ satisfy (3.5) and the system of matrix inequalities

$$
A_{t_{0} \cdots t_{s}} G^{*}+G A_{t_{0} \cdots t_{s}}^{*}+A_{t_{0} \cdots t_{s}} H A_{t_{0} \cdots t_{s}}^{*}+L\left(X_{t_{0} \cdots t_{s}}\right)>0
$$

where

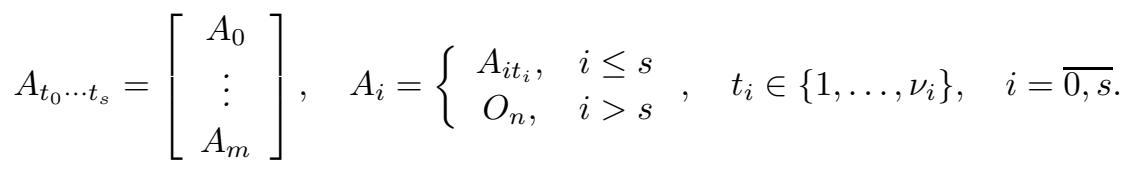

Then for any $p \in \mathcal{P}$, all the eigenvalues of $F(\lambda, p)$ belong to region (3.2).

Proof. We show that for any $p \in \mathcal{P}$,

$$
A(p) G^{*}+G A(p)^{*}+A(p) H A(p)^{*}+L(X(p))>0,
$$

where

$$
A(p)=\left[\begin{array}{c}
A_{0} \\
\vdots \\
A_{m}
\end{array}\right], \quad A_{i}=\left\{\begin{array}{cc}
A_{i}\left(p_{i}\right), & i \leq s \\
O_{n}, & i>s
\end{array}, \quad t_{i} \in\left\{1, \ldots, \nu_{i}\right\}, \quad i=\overline{0, s}\right.
$$


$X(p)$ is a nonnegative linear combination of the matrices $X_{t_{0} \cdots t_{s}}$ satisfied (3.5).

The inequalities (4.2) are ordered by sets of indexes $\left\{t_{0} \cdots t_{s}\right\}$. We specify the indexes $i=0$ and $t_{j} \in\left\{1, \ldots, \nu_{j}\right\}, j \neq 0$, and multiply the $\nu_{0}$ inequalities (4.2) corresponding to the sets of indexes $\left\{1, t_{1} \cdots t_{s}\right\}, \ldots,\left\{\nu_{0}, t_{1} \cdots t_{s}\right\}$ accordingly to $p_{01}, \ldots, p_{0 \nu_{0}}$ and summarize them in consideration of $p_{0}=\left[p_{01}, \ldots, p_{0 \nu_{0}}\right]^{T} \in \mathcal{P}_{\nu_{0}}$. For various combinations of indexes $t_{j} \in\left\{1, \ldots, \nu_{j}\right\}, j \neq 0$, we will perform the same operations. We will use the received inequalities in (4.2) instead of already considered inequalities. Thus, all the inequalities of the given system can be ordered by sets of indexes $\left\{t_{1} \cdots t_{s}\right\}$, where $t_{j} \in\left\{1, \ldots, \nu_{j}\right\}, j=\overline{1, s}$. We will perform similar procedure for every $i=\overline{1, s}$ using the vectors $p_{i}=\left[p_{i 1}, \ldots, p_{i \nu_{i}}\right]^{T} \in \mathcal{P}_{\nu_{i}}$. Finally, we will receive one block inequality and put the corresponding expressions $A_{i}\left(p_{i}\right) H A_{i}\left(p_{i}\right)^{*}$ in the first $s+1$ diagonal blocks. As a result, we will obtain

$$
A(p) G^{*}+G A(p)^{*}+A(p) H A(p)^{*}+S(p)+L(X(p))>0,
$$

where $S(p)$ is a block-diagonal matrix with the diagonal blocks

$$
S_{i}=\left\{\begin{array}{cc}
\sum_{j=1}^{\nu_{i}} p_{i j} A_{i j} H A_{i j}^{*}-A_{i}\left(p_{i}\right) H A_{i}\left(p_{i}\right)^{*}, & i \leq s \\
O_{n}, & i>s
\end{array}, \quad i=\overline{1, m} .\right.
$$

Note that for $i \leq s$, we have

$$
S_{i}=W_{i}\left[\left(P_{i}-p_{i} p_{i}^{T}\right) \otimes H\right] W_{i}^{*}, \quad W_{i}=\left[A_{i 1}, \ldots, A_{i \nu_{i}}\right], \quad P_{i}=\operatorname{diag}\left\{p_{i 1}, \ldots, p_{i \nu_{i}}\right\} .
$$

According to Lemma 4.1, $P_{i} \geq p_{i} p_{i}^{T}$ for $p_{i} \in \mathcal{P}_{i}$. Using the following property of the Kronecker product

$$
P=P^{*} \geq 0, \quad Q=Q^{*} \leq 0 \quad \Longrightarrow \quad P \otimes Q \leq 0,
$$

we get that $S(p) \leq 0$ for $p \in \mathcal{P}$. Hence, the matrix inequality (4.3) holds, and according to Theorem 3.1, all the eigenvalues of the matrix polynomial (4.1) lie in the region (3.2) for any $p \in \mathcal{P}$.

REMARK 4.3. If (4.2) holds for $H=H^{*} \leq 0$, then it is true for $H=O_{n}$ also. Therefore, we can suppose that $H=O_{n}$ always when Theorem 4.2 is used. If $H=H^{*}<0$, then (4.3) is equivalent to the block inequality

$$
\left[\begin{array}{cc}
A(p) G^{*}+G A(p)^{*}+L(X(p)) & A(p) \\
A(p)^{*} & -H^{-1}
\end{array}\right]>0
$$

The inequalities (4.2) are represented in similar form. Dependence on vector parameter $p$ in (4.4) is linear. Therefore, in this case, it is possible to prove Theorem 4.2 without usage of Lemma 4.1. 
Theorem 4.2 can be used for parametrical and interval sets of regular matrix polynomials of the type

$$
F(\lambda, p)=\sum_{i=1}^{\nu} p_{i}\left(A_{0 i}+\lambda A_{1 i}+\cdots+\lambda^{s} A_{s i}\right), \quad p \in \mathcal{P}_{\nu}, \quad \lambda \in \mathbb{C}^{1},
$$

$$
F(\lambda)=A_{0}+\lambda A_{1}+\cdots+\lambda^{s} A_{s}, \quad \underline{A}_{i} \leq A_{i} \leq \bar{A}_{i}, \quad i=\overline{0, s}, \quad \lambda \in \mathbb{C}^{1} .
$$

The set (4.1) is reduced to the form (4.5) when all the vectors $p_{i} \in \mathcal{P}_{\nu_{i}}$ have the same dimension $\nu$ and coincide. In this case, the system (4.2) consists from $\nu$ matrix inequalities. The interval set (4.6) is used most often in applications. It is described in the form (4.1) also. Indeed, for this purpose in (4.1), it is necessary to suppose that

$$
\begin{aligned}
& A_{i}=A_{i}\left(p_{i}\right)=\sum_{j=1}^{\nu_{i}} p_{i j} A_{i j}, \quad A_{i j}=\left\|a_{t \tau}^{i j}\right\|_{t, \tau=1}^{n}, a_{t \tau}^{i j} \in\left\{\underline{a}_{t \tau}^{i}, \bar{a}_{t \tau}^{i}\right\}, \nu_{i}=2^{n^{2}}, \\
& \underline{A}_{i}=\left\|\underline{a}_{t \tau}^{i}\right\|_{t, \tau=1}^{n}, \quad \bar{A}_{i}=\left\|\bar{a}_{t \tau}^{i}\right\|_{t, \tau=1}^{n}, \quad p_{i}=\left[p_{i 1}, \ldots, p_{i \nu_{i}}\right]^{T} \in \mathcal{P}_{\nu_{i}}, \quad i=\overline{0, s} .
\end{aligned}
$$

Then the system (4.2) includes $2^{(s+1) n^{2}}$ matrix inequalities.

Note that, using Lemmas 2.1 and 2.3, we can obtain the generalizations and analogues of Theorem 4.2 for a parametric set of regular matrix functions of the type

$$
F(\lambda, p)=\sum_{i=0}^{m} f_{i}(\lambda) A_{i}\left(p_{i}\right), \quad \operatorname{det} F(\lambda, p) \not \equiv 0, \quad \lambda \in \mathbb{C}^{1}, \quad p=\left[p_{0}^{T}, \ldots, p_{s}^{T}\right]^{T} \in \mathcal{P}
$$

with the corresponding class of regions $\Omega$.

ExAMPLE 4.4. Consider the mechanical system in Figure 4.1 studied in [14]. The system is described by the following differential equations:

$$
\left\{\begin{array}{l}
m_{1} \ddot{x}_{1}+d_{1} \dot{x}_{1}+\left(c_{1}+c_{12}\right) x_{1}-c_{12} x_{2}=0 \\
m_{2} \ddot{x}_{2}+d_{2} \dot{x}_{2}+\left(c_{2}+c_{12}\right) x_{2}-c_{12} x_{1}=u
\end{array}\right.
$$

In [5], the robust stability analysis of the system was carried out with the root-locus inclusion demands in the disk of radius 12 centered at $(-12,0)$ for all admissible interval uncertainty of parameters $\underline{a} \leq a=\left[c_{1}, c_{2}, d_{1}, d_{2}, m_{1}, m_{2}\right] \leq \bar{a}$.

We choose the stability region located in the left half plane:

$$
\Lambda_{2}=\left\{\lambda \in \mathbb{C}^{1}: z(\lambda) \Gamma z^{*}(\lambda)>0\right\}
$$




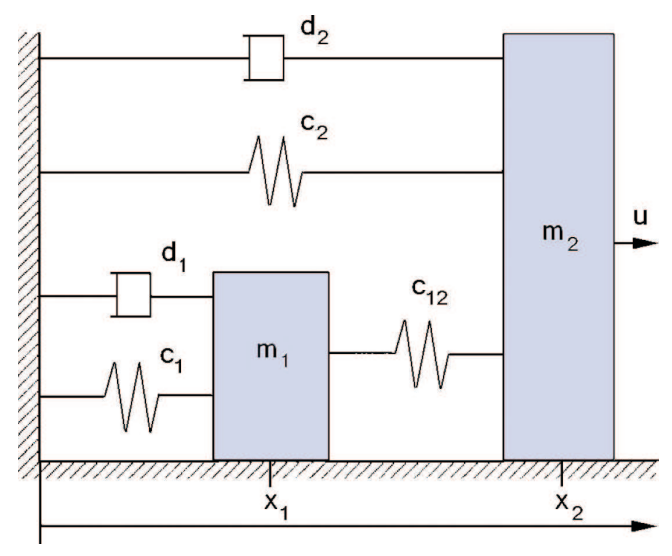

FIG. 4.1. The mechanical system.

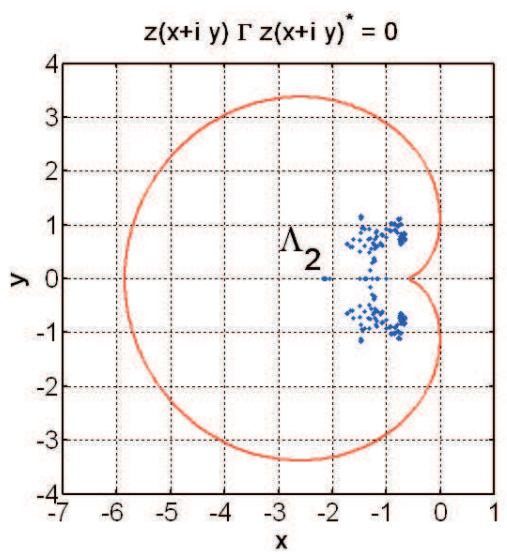

FIG. 4.2. The stability region $\Lambda_{2}$.

where

$$
z(\lambda)=\left[1, \lambda, \lambda^{2}\right], \quad \Gamma=-\left[\begin{array}{ccc}
\frac{9}{16} \alpha^{4} & \frac{7}{4} \alpha^{3} & \frac{9}{4} \alpha^{2} \\
\frac{7}{4} \alpha^{3} & 3 \alpha^{2} & 3 \alpha \\
\frac{9}{4} \alpha^{2} & 3 \alpha & 1
\end{array}\right], \quad \alpha>0
$$

A boundary of $\Lambda_{2}$ is the 4-th order curve called as the Pascal's limacon (see Figure $4.2)$ and defined by the equation

$$
\left[(x+h)^{2}+y^{2}+2 \alpha(x+h)\right]^{2}-\beta^{2}\left[(x+h)^{2}+y^{2}\right]=0,
$$

where $\beta=2 \alpha$ and $h=\alpha / 2$. We set

$$
\alpha=1.3, \quad \underline{a}=[5,6,6,9,2,4], \quad \bar{a}=[6,7,7,10,4,7], \quad c_{12}=1 .
$$

Then the interval matrix polynomial (4.6) corresponding to the open-loop system is given by $F(\lambda)=A_{0}+\lambda A_{1}+\lambda^{2} A_{2}$, where

$$
\begin{gathered}
\underline{A}_{0}=\left[\begin{array}{cc}
6 & -1 \\
-1 & 7
\end{array}\right] \leq A_{0}=\left[\begin{array}{cc}
c_{1}+1 & -1 \\
-1 & c_{2}+1
\end{array}\right] \leq \bar{A}_{0}=\left[\begin{array}{cc}
7 & -1 \\
-1 & 8
\end{array}\right], \\
\underline{A}_{1}=\left[\begin{array}{ll}
6 & 0 \\
0 & 9
\end{array}\right] \leq A_{1}=\left[\begin{array}{cc}
d_{1} & 0 \\
0 & d_{2}
\end{array}\right] \leq \bar{A}_{1}=\left[\begin{array}{cc}
7 & 0 \\
0 & 10
\end{array}\right], \\
\underline{A}_{2}=\left[\begin{array}{ll}
2 & 0 \\
0 & 4
\end{array}\right] \leq A_{2}=\left[\begin{array}{cc}
m_{1} & 0 \\
0 & m_{2}
\end{array}\right] \leq \bar{A}_{2}=\left[\begin{array}{ll}
4 & 0 \\
0 & 7
\end{array}\right] .
\end{gathered}
$$


The system (4.2) to be solved with respect to $X_{t_{0} t_{1} t_{2}}\left(t_{0}, t_{1}, t_{2} \in\{1, \ldots, 4\}\right), G$ and $H$ consists of the 64 matrix inequalities. Assuming that $G=(\underline{A}+\bar{A}) / 2$ and $H=0$, with the help of MATLAB, we find this system feasible. Hence, all the eigenvalues of the stable open-loop mechanical system remain in the domain (4.8) for the specified interval uncertainties. Figure 4.2 shows the roots location of the 64 matrix polynomials $F_{t_{0} t_{1} t_{2}}(\lambda)$ with respect to the Pascal's limacon.

\section{REFERENCES}

[1] I. Gohberg, P. Lancaster, and L. Rodman. Matrix Polynomials. Academic Press, New York, 1982.

[2] A.G. Mazko. Localization of the Spectrum and Stability of Dynamic Systems. Proceedings of the Institute of Mathematics of NAS of Ukraine, Vol. 28. Institute of Mathematics of the NAS of Ukraine, Kiev, 1999 (in Russian).

[3] A.G. Mazko. Matrix Equations, Spectral Problems and Stability of Dynamic Systems. An international book series Stability, Oscillations and Optimization of Systems (Eds. A.A. Martynyuk, P. Borne, and C. Cruz-Hernandez), Vol. 2. Cambridge Scientific Publishers Ltd, Cambridge, 2008.

[4] D. Henrion, O. Bachelier, and M. Sebek. D-stability of polynomial matrices. Internat. J. Control, 74(8):355-361, 2001.

[5] D. Henrion, D. Arzelier, and D. Peaucelle. Positive polynomial matrices and improved LMI robustness conditions. Automatica, 39(8):1479-1485, 2003.

[6] P. Lancaster. Linearization of regular matrix polynomials. Electron. J. Linear Algebra, 17: 21-27, 2008.

[7] N. Higham, D.S. Mackey, and F. Tisseur. The conditioning of linearizations of matrix polynomials. SIAM J. Matrix Anal. Appl., 28(4):1005-1028, 2006.

[8] E.N. Antoniou and S. Vologiannidis. A new family of companion forms of polynomial matrices. Electron. J. Linear Algebra, 11: 78-87, 2004.

[9] V.N. Kublanovskaya. On the spectral problem for polynomial pencils of matrices. Zap. Nauchn. Sem. Leningrad. Otdel. Mat. Inst. Steklov. (LOMI), 80:83-97, 1978.

[10] D. Henrion, M. Sebek, and V. Kucera. Positive polynomials and robust stabilization with fixed-order controllers. IEEE Trans. Automat. Control, 48(7):1178-1186, 2003.

[11] F.R. Gantmaher. The Theory of Matrices. Moskow, Nauka, 1988 (in Russian).

[12] B.T. Polyak and P.S. Shcherbakov. Robust Stability and Control. Moskow, Nauka, 2002 (in Russian).

[13] T.E. Djaferis. Robust Control Design: a Polynomial Approach. Kluver, Boston, 1995.

[14] J. Ackermann. Robust Control. Systems with Uncertain Physical Parameters. Springer Verlag, Berlin, 1993. 